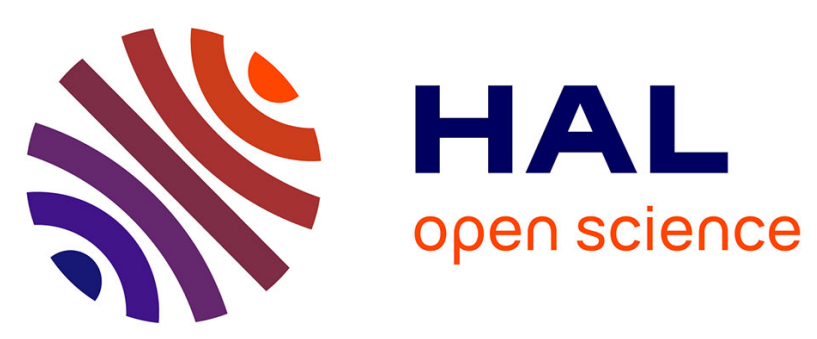

\title{
X-ray absorption spectra of graphene and graphene oxide by full-potential multiple scattering calculations with self-consistent charge density
}

Xu Junqing, Peter Krüger, Calogero R. Natoli, Kuniko Hayakawa, Wu Ziyu, Keisuke Hatada

\section{To cite this version:}

Xu Junqing, Peter Krüger, Calogero R. Natoli, Kuniko Hayakawa, Wu Ziyu, et al.. X-ray absorption spectra of graphene and graphene oxide by full-potential multiple scattering calculations with selfconsistent charge density. Physical Review B: Condensed Matter and Materials Physics (1998-2015), 2015, 92 (12), pp.125408. 10.1103/PhysRevB.92.125408 . hal-01225628

HAL Id: hal-01225628

https://hal-univ-rennes1.archives-ouvertes.fr/hal-01225628

Submitted on 15 Dec 2015

HAL is a multi-disciplinary open access archive for the deposit and dissemination of scientific research documents, whether they are published or not. The documents may come from teaching and research institutions in France or abroad, or from public or private research centers.
L'archive ouverte pluridisciplinaire HAL, est destinée au dépôt et à la diffusion de documents scientifiques de niveau recherche, publiés ou non, émanant des établissements d'enseignement et de recherche français ou étrangers, des laboratoires publics ou privés. 


\title{
X-ray absorption spectra of graphene and graphene oxide by Full Potential Multiple Scattering calculations with self-consistent charge density
}

\author{
Xu Junqing, ${ }^{1}$ Peter Krüger, ${ }^{2}$ Calogero R. Natoli, ${ }^{3}$ Kuniko Hayakawa, ${ }^{3}$ Wu Ziyu,,${ }^{1,4, *}$ and Keisuke Hatada ${ }^{3,5,6, \dagger}$ \\ ${ }^{1}$ National Synchrotron Radiation Laboratory, University of Science and Technology of China, Hefei, Anhui, 230026, China \\ ${ }^{2}$ Graduate School of Advanced Integration Science, Chiba University, Chiba 263-8522, Japan \\ ${ }^{3}$ INFN Laboratori Nazionali di Frascati, Via E Fermi 40, c.p. 13, I-00044 Frascati, Italy \\ ${ }^{4}$ Beijing Synchrotron Radiation Facility, Institute of High Energy Physics, CAS, Beijing 100049, China \\ ${ }^{5}$ Département Matériaux Nanosciences, Institut de Physique de Rennes UMR UR1-CNRS 6251, \\ Université de Rennes 1, F-35042 Rennes Cedex, France \\ ${ }^{6}$ Physics Division, School of Science and Technology, Università di Camerino, \\ via Madonna delle Carceri 9, I-62032 Camerino (MC), Italy
}

(Dated: December 1, 2015)

\begin{abstract}
X-ray absorption near edge structure (XANES) of graphene, graphene oxide and diamond are studied by the recently developed real-space full potential multiple scattering (FPMS) theory with space-filling cells. It is shown how accurate potentials for FPMS can be generated from self-consistent charge densities obtained with other schemes, especially the projector augmented wave method. Compared to standard multiple scattering calculations in the muffin-tin approximation, FPMS gives much better agreement with experiment. The effects of various structural modifications on the graphene spectra are well reproduced. (1) Stacking of graphene layers increases the peak intensity in the higher energy region. (2) The spectrum of the $\mathrm{C}$ atom located at the edge of graphene sheet shows a prominent pre-edge structure. (3) Adsorption of oxygen gives rise to the so-called interlayer-state peak. Moreover, O K-edge spectra of graphene oxide are calculated for three types of bonding, $\mathrm{C}-\mathrm{OH}, \mathrm{C}-\mathrm{O}-\mathrm{C}$ and $\mathrm{C}-\mathrm{O}$, and the proportions of these bondings at $800^{\circ} \mathrm{C}$ are deduced by fitting them to the experimental spectrum.
\end{abstract}

PACS numbers: 78.70.Dm, 61.48.Gh, 71.15.Ap

\section{INTRODUCTION}

Due to its exceptional electronic structure, mechanical strength and high electrical conductivity [1], graphene is expected to become a key material for future nanotechnologies. Graphene oxide is also widely used for reasons of synthesis and chemical stability. X-ray absorption spectroscopy (XAS) and electron energy loss spectroscopy (EELS) are invaluable tools to study the electronic and atomic structure of defects and adsorbates of graphene layers [2-4]. However, because of the existence of various competing structures and the lack of accurate theoretical calculations, the origin of the observed spectral features is generally not well understood yet.

XAS and EELS of graphene has been computed previously using density functional theory (DFT) implemented for ground-state electronic structure calculations $[5,6]$. These methods usually rely on periodic boundary conditions so that the asymptotic behavior of wavefunction may be not reproduced well for low-dimensional systems in particular directions, and use fixed basis sets whose convergence for high energy states may be problematic. Also, the energy-dependent quasiparticle self-energy is replaced by the DFT ground exchangecorrelation potential $\left(V_{x c}\right)$. Concerning these problems, the multiple scattering method has clear advantages, be-

\footnotetext{
* † Deceased 23 March 2015.

$\dagger$ keisuke.hatada@univ-rennes1.fr
}

cause the single-particle Schrodinger equation is solved with the exact numerical basis functions for each energy. As a consequence, basis function convergence is not an issue and inclusion of energy dependent self-energies is straightforward. Moreover, the multiple scattering method is easily implemented in reciprocal-space, realspace and mixtures thereof, which makes it a method of choice for low-dimensional and nanostructured systems.

Conventional multiple scattering theory relies on the Muffin-Tin (MT) approximation where the potential is assumed spherically symmetric in each atomic (MT) sphere and constant outside the spheres, that is in the so-called interstitial region. The MT approximation is quite poor for graphene-like systems for the following reasons: (i) The charge density of the C-C $\sigma$ and $\pi$ bonding is highly anisotropic. (ii) For light elements such as Carbon, atomic potential scattering is rather weak and thus scattering of the interstitial potential is comparatively strong. Hence full potential (FP) corrections are important. (iii) The available energy range for x-ray absorption fine structure of light element is limited to a few tens of $\mathrm{eV}$. This near-edge region shows rich spectral features due to strong scattering effects and the peaks are sharp because of long core hole lifetimes. Therefore, FP and SCF are necessary to increase the precision of the calculations.

Here we report XANES calculations of ideal and defective graphene, graphene oxide and diamond, using the real-space full potential multiple scattering (FPMS) theory with arbitrarily truncated space-filling cells $[7,8]$. 
We also present a method to generate all-electron potentials for FPMS from accurate charge-densities provided by other electronic structure codes, in particular projector augmented wave (PAW) methods [9]. This allows us to access the importance of FP corrections and selfconsistency in multiple scattering calculation of C K-edge XANES. The results are in good agreement with experiment and shed light on the origin of some controversial features of the graphene XANES.

\section{THEORY}

\section{A. FPMS method}

There have been many attempts to extend the MS theory to the FP level [10-15]. Here we give a brief summary of our FPMS method developed previously by some of us [7]. The real-space FPMS theory with spacefilling cells used here is valid both for continuum and bound states and contains only one truncation parameter - lmax, the maximum angular momentum of spherical wave basis. Space is partitioned by non-overlapping space-filling cells or Voronoi polyhedra. When a Voronoi polyhedron does not contain any atom or is in the interstitial region but still contains charge density, it is called empty cell (EC). Local Schrodinger equation is solved without the limit of the geometrical shape of the potential since the potential is not expanded in spherical harmonics. The absorption cross section is given by

$$
\begin{aligned}
& \sigma(\omega)=-8 \pi \alpha \hbar \omega \\
& \quad \times \sum_{m_{c}} \operatorname{Im} \int\left\langle\phi_{L_{c}}^{c}(\vec{r})|\hat{\varepsilon} \cdot \vec{r}| G\left(\vec{r}, \overrightarrow{r^{\prime}} ; E\right)\left|\hat{\varepsilon} \cdot \overrightarrow{r^{\prime}}\right| \phi_{L_{c}}^{c}(\vec{r})\right\rangle d \vec{r} d \overrightarrow{r^{\prime}},
\end{aligned}
$$

where $\phi_{L_{c}}^{c}$ is the initial core state with $L_{c} \equiv\left(l_{c}, m_{c}\right)$ representing the orbital angular momentum and magnetic quantum number, $\omega$ is the frequency of incident light, $\alpha$ is fine structure constant, $\hat{\varepsilon}$ is the electric field direction of incident light and $E$ is the energy of final state. Green's function can be written as

$$
\begin{aligned}
G\left(\overrightarrow{r_{i}}, \overrightarrow{r_{j}^{\prime}} ; E\right)= & \left\langle\underline{\Phi}\left(\overrightarrow{r_{i}}\right)\left|\left([I-G T]^{-1} G\right)^{i j}\right| \underline{\Phi}\left(\overrightarrow{r_{j}^{\prime}}\right)\right\rangle \\
& -\delta_{i j}\left\langle\underline{\Phi}\left(\overrightarrow{r_{<}}\right) \mid \Psi\left(\overrightarrow{r_{>}}\right)\right\rangle,
\end{aligned}
$$

where $\overrightarrow{r_{i}}$ is the coordinate with respect to the center of scattering site $i$. $T$ and $G$ on the right hand side of the equation are the matrix of transition operators of scattering sites and KKR real-space structure factors, respectively. $\underline{\Phi}_{L} \equiv \sum_{L^{\prime}}(\tilde{E})_{L L^{\prime}}^{-1} \Phi_{L^{\prime}}$ where $\Phi$ is the solution of local Schrodinger equation which behaves as the first kind of spherical Bessel function at the origin and $\tilde{E}$ matrix can be computed using values on surface of the cell. $\Psi$ is the solution of local Schrodinger equation irregular at the origin which matches smoothly to spherical Hankel function at the cell boundary. $r_{<}\left(r_{>}\right)$is the smaller (bigger) one of $r$ and $r^{\prime}$.

\section{B. FPMS potentials from PAW charge densities}

Plane-wave codes using the PAW method are popular because of high accuracy and computational efficiency for total energy DFT calculations. In PAW, the all-electron Kohn-Sham potential is replaced by a pseudo-potential inside the augmentation spheres around the nuclei. This pseudo-potential coincides with the all-electron potential only in the space outside the augmentation sphere. However, for XANES calculations the all-electron potential is required in all space, especially near the nuclei where the core-orbital has a large amplitude. We now present a method for reconstructing the all-electron potential in the augmentation spheres from the all-electron charge density, which is commonly provided in PAW methods, such as the Vienna Ab initio Simulation Package (VASP) [16] that we have used here. As the calculation of a local or semi-local exchange correlation potential from the charge density is straightforward, we only explain how to obtain the all-electron Hartree potential.

The mathematical problem is to find the regular solution of the Poisson equation inside an (augmentation) sphere $S$ of radius $r_{c}$, with Dirichlet boundary condition on the sphere, i.e., we want to solve

$$
\nabla^{2} V(\vec{r})=-4 \pi \rho(\vec{r})
$$

for $r<r_{c}$, given the charge density $\rho$ for all points inside $S$, and the potential $V$ on the surface $\left(r=r_{c}\right)$. We introduce the function

$$
W(\vec{r}) \equiv \int_{S} \frac{\rho\left(\overrightarrow{r^{\prime}}\right)}{\left|\vec{r}-\overrightarrow{r^{\prime}}\right|} d \overrightarrow{r^{\prime}}
$$

which satisfies the Poisson equation, but in general not the boundary condition. $W(\vec{r})$ can easily be calculated in a spherical harmonic basis

$$
\begin{gathered}
W(r, \hat{r})=\sum_{L} \omega_{L}(r) Y_{L}(\hat{r}) \\
\omega_{L}(r)=\int_{0}^{r_{c}} \frac{r_{<}^{l}}{r_{>}^{l+1}} \rho_{L}\left(r^{\prime}\right) 4 \pi{r^{\prime}}^{2} d r^{\prime}, \\
\rho_{L}(r)=\int Y_{L}^{*}(\hat{r}) \rho(r, \hat{r}) d \hat{r} .
\end{gathered}
$$

where $\hat{r}=\vec{r} / r$. The angular integrals can be performed very accurately on a Lebedev mesh [17]. The general solution of the Poisson equation is the sum of a particular solution, such as $W$, and the general solution of the corresponding homogeneous (i.e. Laplace) equation. The latter solution is $\sum_{L} C_{L} r^{l} Y_{L}(\hat{r})$ for arbitrary coefficients $C_{L}$. By choosing $C_{L}=\left[v_{L}\left(r_{c}\right)-\omega_{L}\left(r_{c}\right)\right] / r_{c}^{l}$, where

$$
v_{L}(r)=\int Y_{L}^{*}(\hat{r}) V(r, \hat{r}) d \hat{r}
$$

the boundary conditions are satisfied, and so the final solution is given by

$$
V(r, \hat{r})=\sum_{L}\left(\omega_{L}(r)+\left[v_{L}\left(r_{c}\right)-\omega_{L}\left(r_{c}\right)\right] \frac{r^{l}}{r_{c}^{l}}\right) Y_{L}(\hat{r}) .
$$




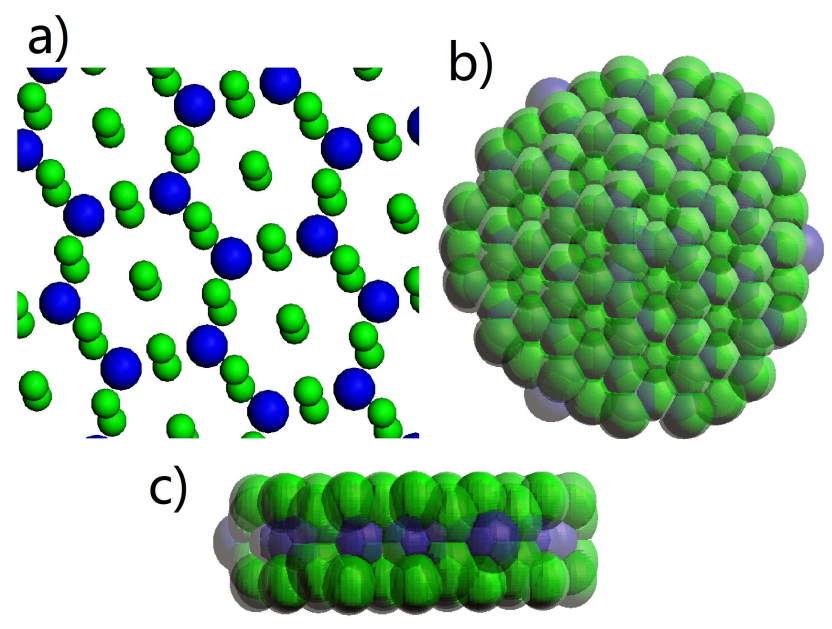

FIG. 1. (a) Positions of carbon atoms (blue ball) and ECs - empty cells (green ball) for graphene. (b) and (c) show schematic diagrams of the graphene cluster with space filling cells viewed along $\mathrm{z}$ and $\mathrm{y}$ axis, respectively. The blue cell represents a carbon atom, and the green cell is EC.

This method has been implemented in a program "VASP2MS" which reads the charge density $\rho$ and the Hartree (pseudo-)potential (for $V\left(r_{c}, \hat{r}\right)$ ) provided by the VASP code and calculates the all-electron Hartree potential in all space for use in the FPMS code. In practice the following VASP files are used: AECCARO, 1, 2 which contain the core $(0)$, non-SCF valence $(1)$ and SCF valence (2) charge density, respectively. LOCPOT which contains the Hartree pseudopotential inside the atomic spheres and true potential in the rest of the unit cell. The data in these files is stored on a regular $3 \mathrm{D}$ grid of the unit cell. Further, RADCHGC and RADCHGV contain the core and valence charge density, expanded in spherical harmonics and stored on radial grids around each nucleus. In "VASP2MS", the VASP Hartree potential (in LOCPOT) is interpolated onto the radial meshes used in FPMS, in the region outside the augmentation spheres. The allelectron charge density $\rho$ is interpolated onto the FPMS mesh everywhere in the cluster. Then, the method described above is applied to find the all-electron Hartree potential inside the augmentation spheres. Finally the exchange-correlation part of the potential is calculated from $\rho$ and added to the Hartree potential.

\section{Computational details}

The C K-edge XANES spectra are calculated with the real-space multiple scattering method using the following forms of the potential. 1) non-SCF-FP: a full-potential calculation without self-consistency. The potential is calculated directly in the FPMS code and corresponds to the superposition of atomic charge densities. 2) SCFFP: a self-consistent FP calculation, where the potential has been reconstructed from the charge density obtained

\begin{tabular}{lcccc}
\hline \hline $\begin{array}{c}\text { System } \\
\text { and type }\end{array}$ & $\mathrm{x}$ & $\mathrm{y}$ & $\mathrm{z}$ & $\mathrm{r}$ \\
\hline $\begin{array}{l}\text { 1. Graphene } \\
\text { Carbon }\end{array}$ & 1.424 & 0 & 0 & 0.95 \\
Carbon & 2.848 & 0 & 0 & 0.95 \\
EC & 0 & 0 & \pm 0.7 & 1.05 \\
EC & 1.068 & \pm 0.6166101 & 1.2 & 0.9 \\
EC & 1.068 & \pm 0.6166101 & -1.2 & 0.9 \\
EC & 2.136 & 0 & \pm 1.2 & 0.9 \\
2. Diamond & & & & \\
Carbon & \pm 0.445875 & \pm 0.445875 & \pm 0.445875 & 0.89175 \\
EC & \pm 1.337625 & \pm 1.337625 & \pm 1.337625 & 0.89175 \\
\hline \hline
\end{tabular}

TABLE I. Cartesian coordinates and radius of polyhedra of atoms and ECs in the unitcell of graphene and diamond. All data are in the unit of $\AA$.

by VASP code with the PAW [9] method. 3) SCF-MT: the same as SCF-FP except that the muffin-tin (MT) approximation is applied.

The graphene layer is taken as the xy plane and the $\mathrm{x}$ axis along a carbon-carbon bond. The absorption cross section for light polarized along $a=x, y, z$ is denoted $\sigma_{a}$, $\sigma=\left(\sigma_{z}+\sigma_{x}+\sigma_{y}\right) / 3$ is the unpolarized spectrum, and we define $\sigma_{x-y}=\left(\sigma_{x}+\sigma_{y}\right) / 2$ as the in-plane $(z=0)$ cross section. For systems with $\mathrm{C} 3 \mathrm{v}$ point symmetry or higher, such as core-excited graphene which has D3h point symmetry, we have $\sigma_{x}=\sigma_{y}$. For an angle $\alpha$ between electric field and xy plane, the absorption cross-section is $\sigma_{\alpha}=\sigma_{z} \sin ^{2} \alpha+\sigma_{x} \cos ^{2} \alpha$.

Table I lists positions and spherical radii of atoms and ECs in the unit cell of graphene and diamond. Figure 1 is a schematic representation of the space partitioning used for graphene. The number of ECs is sufficient as we have checked by adding two more EC layers which did not change the XANES results. For the optical potential we take the Hedin-Lundqvist (HL) form [18] whose energydependent imaginary part accounts for damping effects through plasmon loss, with an additional constant imaginary potential accounting for the finite core-hole lifetime and leading to Lorentzian broadening. The lifetime broadening is $0.1 \mathrm{eV}[19,20]$ for $\mathrm{C}$ K-edge and $0.15 \mathrm{eV}$ for O K-edge [20, 21], but in case of graphene, the value

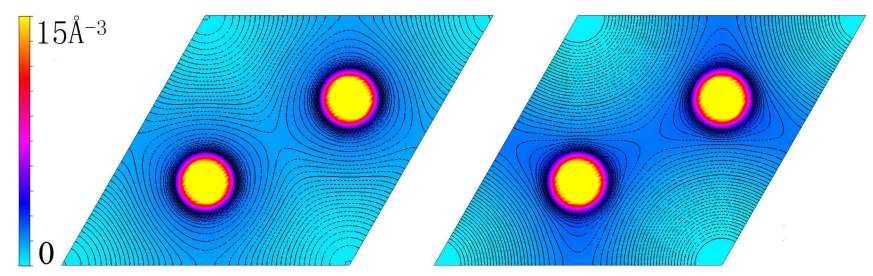

FIG. 2. Charge density in the xy-plane $(z=0)$ of the graphene unit cell from the non-SCF FPMS (left) calculation and the SCF (VASP) calculation (right). The unit of charge density is $\AA^{-3}$. Contour lines are plotted for values $F_{i}=5^{(-1+0.1 i)}$ $\AA^{-3}$, where $i=0,1 \ldots 20$, from the corner to the region around carbon atoms. 


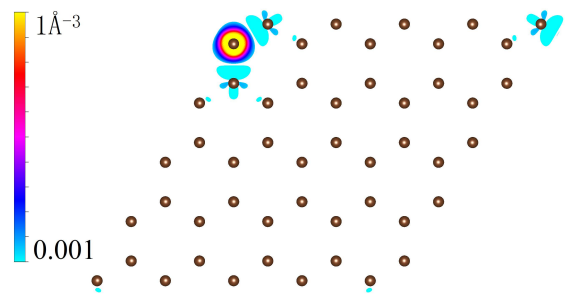

FIG. 3. Valence charge density difference in graphene xyplane, induced by a core-hole in the left-top C-atom of the $5 \times 5 \times 1$ supercell. The unit of charge density is $\AA^{-3}$. White corresponds to the value 0 and Carbon atoms is indicated by brown balls.

$0.2 \mathrm{eV}$ was also reported [22, 23]. In order to better see the spectral fine structure at high energy, the imaginary part of the HL potential has been omitted in a few cases (Figs. 5, 8 and 16). Moreover, the spectra are Gaussian broadened by $0.4 \mathrm{eV}$ (graphene) and 0.3 $\mathrm{eV}$ (diamond) in order to account for experimental effects such as instrumental errors, structural uncertainties, vibration, etc. From the convergence test, we take $\operatorname{lmax}=3$.

In Fig. 2 the effect of self-consistency on the groundstate charge density of graphene is shown for the $z=0$ plane. Differences between SCF and non-SCF calculations are clearly visible, but the charge redistribution appears to be rather small.

Core-hole effects are treated using the so-called finalstate rule (FSR), that is, in the final state the orbitals are relaxed around the full core-hole. In non-SCF-FP, this relaxation is calculated self-consistently but only at the atomic level. In SCF-FP, orbital relaxation effects beyond the absorbing atom are also taken into account through a VASP supercell calculation which yields the self-consistent FSR charge density and electrostatic potential for the bulk material. In this case, the core electron is put into the lowest unoccupied molecular orbital and the frozen-core approximation is applied, i.e., only valence electrons are relaxed. In order to make the interaction between periodic images of the core-hole negligible, a large supercell (SC) must be used. The converged size of $\mathrm{SC}$ is found to be $4 \times 4 \times 1$ for graphene and few-layer graphene (FLG), and $4 \times 4 \times 4$ for diamond. However, for safety, bigger SCs are used in this work as shown in Table II.

Figure 3 shows the distribution of the differential allelectron valence charge densities in xy plane, which are

\begin{tabular}{ccccc}
\hline \hline \multirow{2}{*}{ parameter } & \multicolumn{2}{c}{ graphene } & \multicolumn{2}{c}{ diamond } \\
\cline { 2 - 5 } & $\begin{array}{c}\text { core- } \\
\text { hole }\end{array}$ & $\begin{array}{c}\text { no core- } \\
\text { hole }\end{array}$ & $\begin{array}{c}\text { core- } \\
\text { hole }\end{array}$ & $\begin{array}{c}\text { no core- } \\
\text { hole }\end{array}$ \\
\hline $\mathrm{k}$ points & $3 \times 3 \times 1$ & $15 \times 15 \times 1$ & 1 & $11 \times 11 \times 11$ \\
Ecut $(\mathrm{eV})$ & 680 & 1100 & 680 & 1100 \\
supercell & $5 \times 5 \times 1$ & 1 & $5 \times 5 \times 5$ & 1 \\
\hline \hline
\end{tabular}

TABLE II. Parameters used in VASP calculations. the differences between the densities of a $5 \times 5 \times 1 \mathrm{SC}$ of graphene with a core-hole, and that of the ground-state graphene. It is seen that charge density redistribution is negligible for points further than $2.5 \AA$ from the corehole.

In the DFT VASP calculation, the gradient corrected exchange-correlation functional PW91 [24] has been used but the local density approximation gives virtually identical results as we have checked. K-point sampling in the Brillouin zone and plane wave cut-off energy (Ecut) are given in Table II. For the SC dimension perpendicular to the layer, we took $15 \AA$ such that the interaction between periodic images of the graphene layer is negligible. When the VASP potentials are imported to the real-space cluster calculation, the final state potential is used only for atoms not further away from the absorbing site than some distance $R$, of the order of half the SC dimension. For atoms beyond $R$, the ground-state VASP potential is used. In this way the periodic repetition of core-holes, which is an artefact of $\mathrm{k}$-space methods (such as VASP) is avoided and convergence of the final results as a function of VASP SC size is very efficient.

\section{XANES OF GRAPHENE}

From Ref. [25] and [26], we have summarized the representative peaks of graphene and FLG in Table III. The calculated values correspond to SCF-FP calculation with a cluster size of $30 \AA$. The theoretical energy scale has been rigidly shifted such as to align peak $\mathrm{A}$ with experiment $(285.5 \mathrm{eV})$. Peaks A, B and B2 were identified as $\pi^{*}$ resonance, $\sigma^{*}$ resonance and excitonic-state $[27,28]$ peak, respectively.

In Fig. 4 the experimental spectra [25, 26] for two light polarizations are shown along with the present calculations done in different potential approximations. It is obvious that the FP calculations (non-SCF-FP or SCFFP) agree much better with experiment than the muffintin calculation (SCF-MT). The differences between nonSCF-FP and SCF-FP spectra are small, indicating that self-consistency affects XANES much less than FP cor-

\begin{tabular}{|c|c|c|c|c|c|}
\hline \multicolumn{2}{|c|}{$\begin{array}{r}\sigma_{z} \\
\text { energy }(\mathrm{eV})\end{array}$} & \multirow{2}{*}{ symbol } & \multicolumn{2}{|c|}{$\begin{array}{r}\sigma_{x} \\
\text { energy }(\mathrm{eV})\end{array}$} & \multirow{2}{*}{ symbol } \\
\hline cal & $\exp$ & & cal & $\exp$ & \\
\hline 283.7 & 283.7 & $\mathrm{~A} 2$ & - & 291.7 & B2 \\
\hline 285.5 & 285.5 & $\mathrm{~A}$ & 292.5 & 292.6 & $\mathrm{~B}$ \\
\hline- & 288.2 & K & 297.1 & 297.8 & $\mathrm{D}$ \\
\hline 294.5 & 294.0 & $\mathrm{C}$ & 301.5 & 301.8 & $\mathrm{E}$ \\
\hline 307.7 & 308.0 & $\mathrm{G}$ & 307.0 & 306.8 & $\mathrm{~F}$ \\
\hline- & 315.5 & $\mathrm{H}$ & 327.0 & 327.5 & I \\
\hline
\end{tabular}

TABLE III. Peak positions of calculated XANES of graphene with $30 \AA$ size in SCF-FP case and experimental XANES of graphene and FLG. Peak $\mathrm{H}$ only exists in the experiment of FLG. "-" represents not clear or not being reproduced in calculations. 

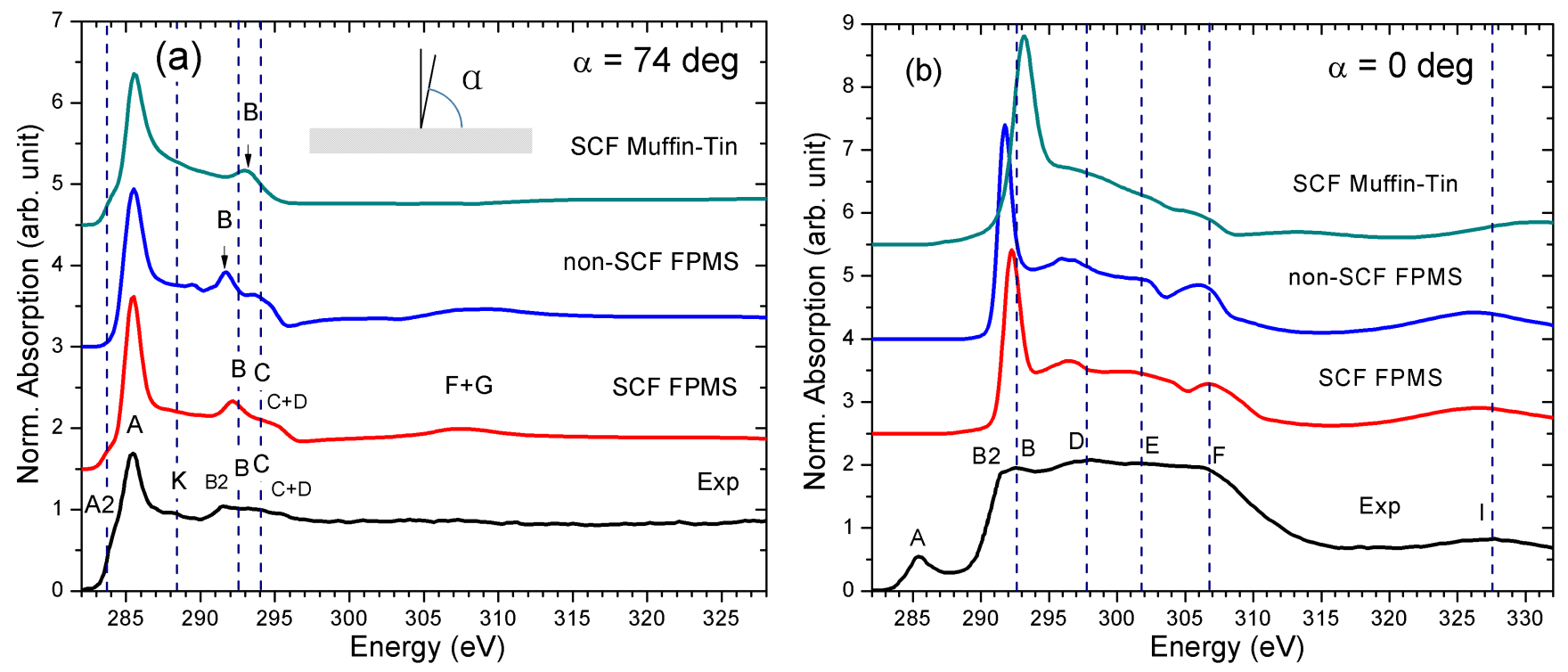

FIG. 4. Comparison between calculated polarized C K-edge XANES of graphene with a cluster of radius $30 \AA$ and experiments $[25,26] . \alpha$ is the angle between electric field and xy plane. Since $\alpha=74$ deg in left panel, $\sigma \approx 0.924 \sigma_{z}+0.076 \sigma_{x}$ (see Sec. II C). The reason why peak A arises in right panel despite the fact that $\alpha=0 \mathrm{deg}$ is discussed in the text of Sec. III. Dashed vertical lines show the peak positions of experiments. SCF-FP, non-SCF-FP and SCF-MT are defined in Sec. II C. (non-)SCF represents using (non-)self-consistent potential. While FP and MT represent full potential and Muffin-Tin version of multiple scattering theory, respectively.

rections. The peak positions of non-SCF-FP and SCFFP spectra differ by about $1 \mathrm{eV}$ and SCF-FP shows better agreement with experiment. Indeed, for out-of-plane polarization $\left(\alpha=74^{\circ}\right.$, Fig. $\left.4(\mathrm{a})\right)$ the SCF-FP calculation agrees very well with the data. However, a peak near $307.5 \mathrm{eV}$, labeled as " $\mathrm{F}+\mathrm{G}$ ", appears in the SCF-FP result but it is absent or faint in experiment, see Fig. 4(a).

For in-plane polarization $\left(\alpha=0^{\circ}\right.$, Fig. 4(b)) there is some systematic disagreement between experiment and all calculations, namely overestimation of peak B intensity and absence of peaks A and B2. The presence of peak $\mathrm{A}$ in the experimental data of Fig. 4(b) is very surprising, since peak A was identified in the $\sigma_{z}$ spectrum (Fig. $4(\mathrm{a}))$ as the $\pi^{*}$ resonance, which should exactly vanish for in-plane $\left(\alpha=0^{\circ}\right)$ polarization (as it does in the calculations). This observation strongly suggests that some out-of-plane signal $\left(\sigma_{z}\right)$ is mixed into the experimental spectra of Fig. 4(b). The reasons may be a misalignment in the experiment, or more likely, the fact that the graphene layer is not perfectly flat [29], such that locally the electric field cannot be parallel to the graphene plane everywhere.

The leading low energy peaks of the calculated spectra (peak A in Fig. 4(a) and peak B in Fig. 4(b)) are too intense, which indicates that charge relaxation around the core-hole is overestimated. This might be due to the residual self-interaction in DFT [30]. Further, the excitonic-state - peak B2 separated from peak B by about $1.0 \mathrm{eV}$ in the experiment is not reproduced in the calculations even if the gaussian broadening is reduced to $0.2 \mathrm{eV}$. The theoretical problems with the leading peaks
(B, B2) are likely related to the frozen-core approximation and to dynamical screening effects, i.e., the energy dependence of the complex self-energy [31, 32]. The nonlocality of the particle-hole exchange interaction [33] is a further issue. It should be noted that the poor descrip-

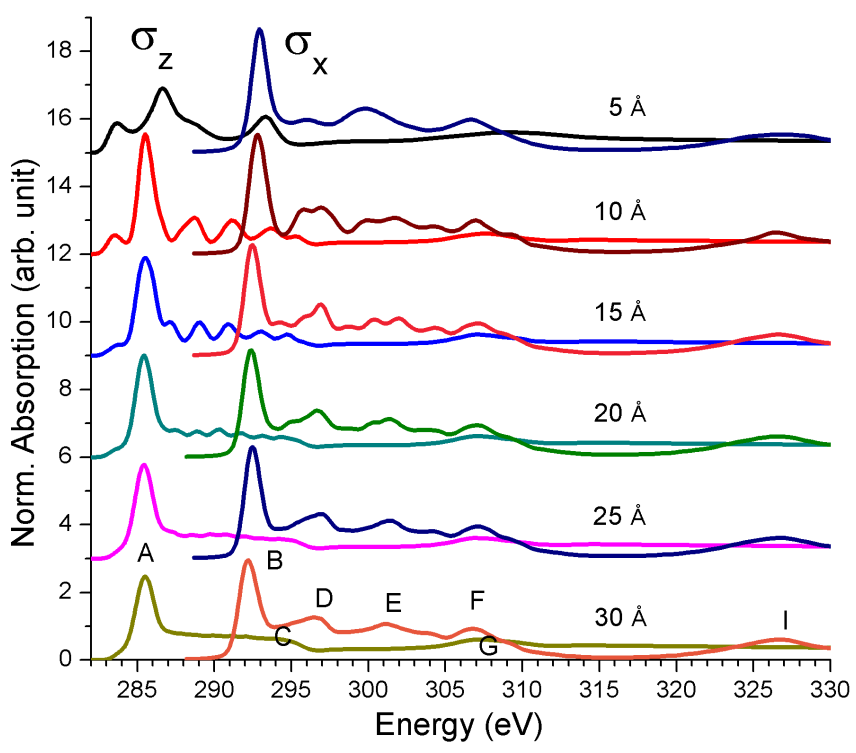

FIG. 5. Calculated XANES in SCF-FP case with different sizes - radii of clusters whose center is the absorbing atom. $\sigma_{z(x)}$ is polarized absorption cross section with electric field along $\mathrm{z}(\mathrm{x})$ axis. 
tion of the leading peaks is a common problem for K-edge XANES of light elements and could so far not be cured by any ab initio method we are aware of, including the Bethe-Salpeter-Equation approach [34].

Furthermore, in the experiment in Fig. 4(a), two weak peaks, a pre-edge one - A2 and peak $\mathrm{K}$, were observed near 284 and $288 \mathrm{eV}$, respectively. In Ref. [25], peak A2 was attributed to a state about $0.8 \mathrm{eV}$ below $\pi^{*}$, which was mentioned in a rather old density of state (DOS) calculation of graphene [35]. However, in more recent DOS calculations [36-38], there does not exist any unoccupied state lower than $\pi^{*}$ state. We therefore believe that there is no such state in perfect graphene, and that the observation of peak A2 in Ref. [25] was due to a structural imperfection. In Sec. III C we show that edge atoms can produce a feature similar to peak A2.

The origin of peak $\mathrm{K}$ has been contentious, and alternative descriptions ascribe it to residual functionalization $[39,40]$, especially the bonding between carbon and oxygen, or a free-electron-like interlayer-state [25, 41]. In our SCF-FP result of XANES of the graphene cluster of radius $30 \AA$, for out-of-plane polarization $\left(\alpha=74^{\circ}\right.$, Fig. 4(a)), there does not exist any distinct peak near 288 $\mathrm{eV}$. Therefore, peak K probably will not arise in XANES of ideal graphene. Possible origins are discussed at the end of this section and Sec. IV A.

\section{A. Cluster size dependence}

In the following, the theoretical spectra correspond to the SCF-FP method, unless stated otherwise. Figure 5 shows the cluster size dependence of the calculated spectra. Good convergence is achieved for a cluster radius of about $20 \AA$, except for some very fine features. Convergence is faster on the high energy side of the spectrum, as it is usually observed in real-space calculations for states above Fermi level.

We have found that the pre-edge peak - A2 and the oscillatory features between peaks A and B, can appear for small clusters, but as the cluster size increases, they become weaker. For a cluster radius of $30 \AA$, the features between peak $\mathrm{A}$ and $\mathrm{B}$ are gone. Therefore, finite size and vacancies in the graphene sheet may contribute to peak A2 and K. However, we can expect these peaks to be negligible for graphene of high structural quality, that is to say, perfectly flat sheets of several $\mu m$ size or bigger with low defect concentration.

\section{B. Layer effects}

In this section, XANES calculations of 2-layer graphene (2LG), 3-layer graphene (3LG) and graphite are reported. The clusters radius is $15 \AA$ and the distance between layers is $3.4 \AA$. We consider Bernal stacking whose order is $1212 \ldots$ [1] as shown in the left panel of Fig. 6. Each layer has two types of carbon atoms - A and

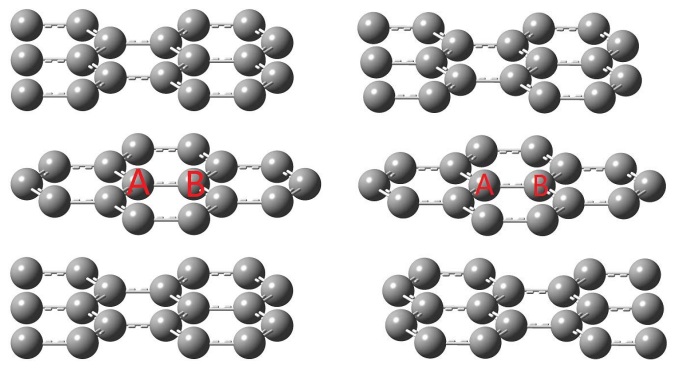

FIG. 6. Schematic diagram of Bernal (left) and rhombohedral (right) stacking of graphene layers.

B, giving a total of two types in both $2 \mathrm{LG}$ and graphite and four types in 3LG. The spectra of the different types are summed for comparison with experiment. The cluster potential is constructed using the strategy outlined above. Explicitly, for sites not further than 6 A from the absorber and in the same layer, the core-excited (supercell) graphene VASP potential is used. For all other sites, the graphite VASP ground-state potential is used.

In order to check that this way to construct the cluster potential is reliable, we have compared it with another strategy, where both VASP potentials of core-excited (SC) 3LG and ground-state 3LG are used. We find that both strategies give very similar results (not shown) which indicates that charge density of a graphene layer is not much affected by the presence of other layers. Consistently, the spectral differences (not shown) between the inequivalent sites A, B is also small.

Apart from Bernal stacking, other stackings have been reported for multilayer graphene, most importantly rhombohedral stacking whose order is 123123... [1] (see

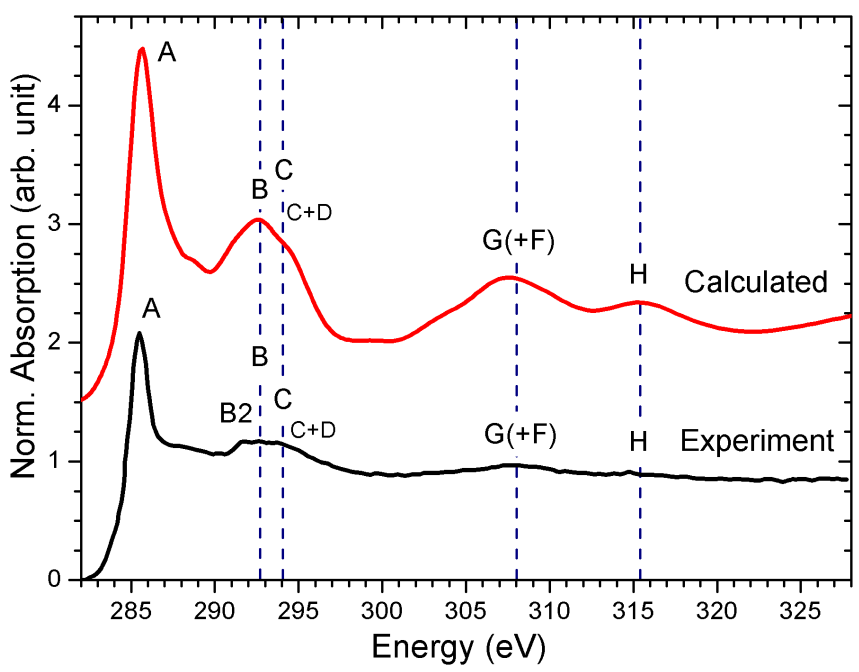

FIG. 7. Comparisons between calculated polarized C K-edge XANES of $3 \mathrm{LG}$ with a cluster whose size is $15 \AA$ and the experiment [25]. The angle between electric field and xy plane is also $74 \mathrm{deg}$. To achieve better agreement with the experiment, we used $0.7 \mathrm{eV}$ Gaussian broadening. 


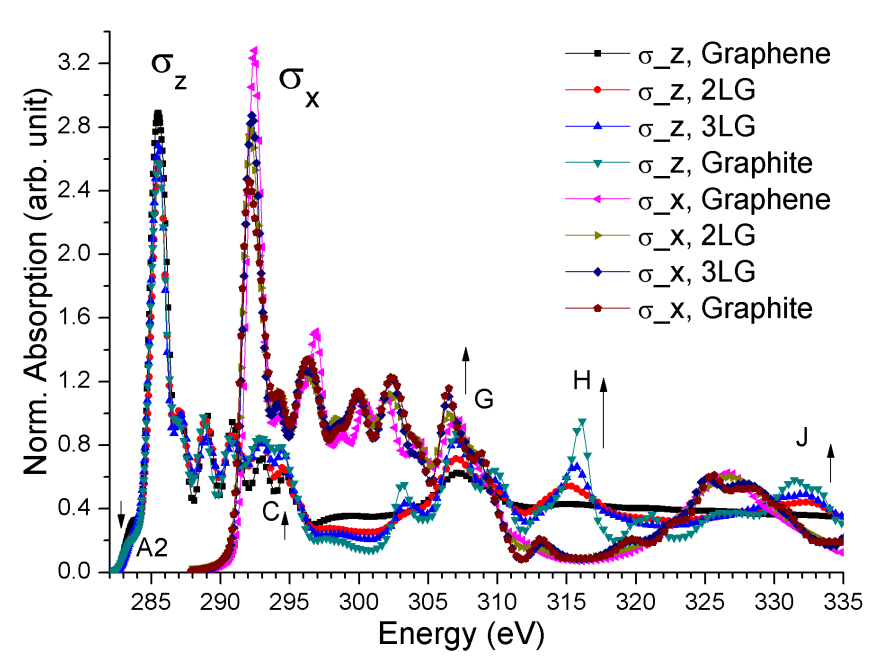

FIG. 8. Calculated XANES of graphene, 2LG, 3LG and Graphite. $\sigma_{-} \mathrm{z}(\mathrm{x})$ represents $\sigma_{z(x)}$. Arrow represents the variation trend of peak intensity as layer number increases.

the right panel of Fig. 6). We have calculated the $\mathrm{C}$ K-edge XANES of 3LG in rhombohedral stacking, but found only tiny differences from Bernal stacking. The differences appear to be too small for an experimental determination of the stacking sequence from the XANES results.

The XANES of 3LG are shown in Fig. 7. The calculated peak intensities are systematically larger than in the data, but the positions of the peaks and their relative intensities are very well reproduced. In the calculated spectrum, a weak feature can be seen at $289 \mathrm{eV}$, close to the peak K energy. We note that the peak in the calculation is due to the finite cluster radius ( $15 \AA$ ) in the xyplane. In Fig. 8, graphene, 2LG, 3LG and graphite are compared. When the number of layers goes up, peaks G, $\mathrm{H}$ and $\mathrm{J}[42]$ increase quite strongly and peak C slightly, whereas peak A2 decreases somewhat. These tendencies agree well with experiment $[25,26]$. Moreover, the oscillatory features near $288.5 \mathrm{eV}$ remain unchanged, which indicates that the effects of stacking of graphene layers on peak $\mathrm{K}$ is weak.

\section{Edge effects}

One-dimensional structures such as graphene ribbons are interesting for potential applications. The reduced dimensionality and the presence of the edge atoms can strongly modify the electronic and transport properties of graphene. The local electronic structure at the edge of a graphene has been measured by X-ray absorption microscopy [43] and EELS [44, 45]. Here we study XANES of a zigzag-type edge terminated by hydrogen, and focus on several particular kinds of carbon atoms which are colored pink and labelled by numbers as shown in Fig. 9. The spectra are calculated using a cluster of the semi-

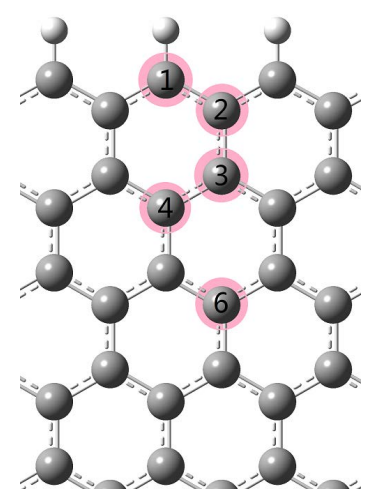

FIG. 9. Schematic diagram of the graphene zigzag edge.

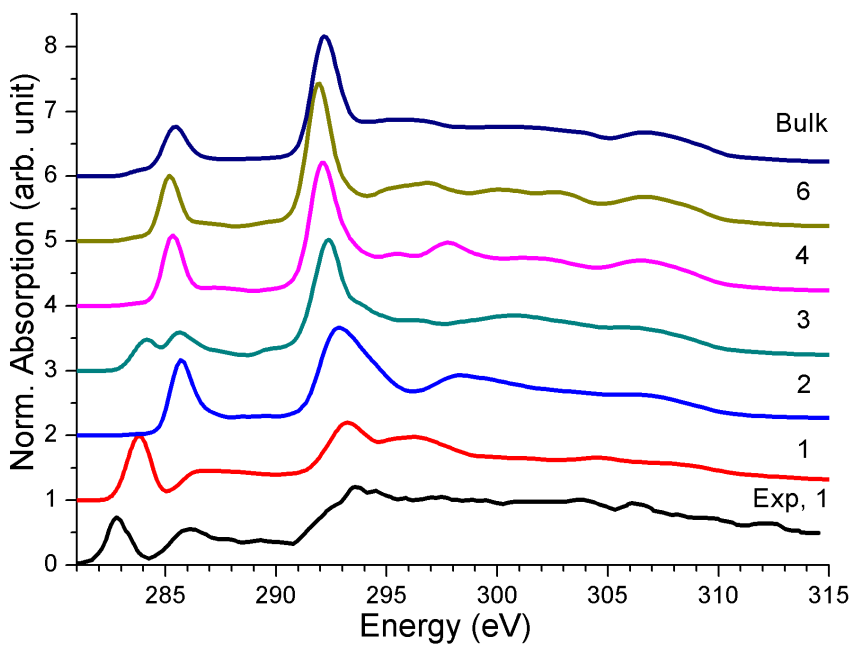

FIG. 10. Calculated unpolarized C K-edge XANES of individual $\mathrm{C}$ atoms at or near the graphene zigzag edge, in comparison with calculated XANES of infinite graphene (labelled by "Bulk") and experimental EELS spectrum of the individual outermost $\mathrm{C}$ atom at the edge taken from Ref. [44]. Each number corresponds to the $\mathrm{C}$ atom which is labelled by the same number as in Fig. 9.

infinite graphene zigzag edge of radius of $25 \AA$ around the absorber. Atomic positions at the edge are relaxed in a VASP calculation with a $1 \times 22 \mathrm{SC}$ containing 1 hydrogen and 22 carbon atoms along the axis perpendicular to the edge. The final state potential is generated as follows. Atomic and EC sites far from the edge by about $12 \AA$, the graphene ground-state potential is used. For sites near the edge, FSR potentials have been generated using a $4 \times 16$ SC terminated by hydrogen on both sides ( with $8 \mathrm{H}$ and $64 \mathrm{C}$ atoms) and one core-hole on the absorbing site.

Figure 10 shows calculated unpolarized C K-edge XANES of individual $\mathrm{C}$ atoms at or near the graphene zigzag edge, in comparison with calculated XANES of infinite graphene and experimental EELS spectrum of the individual outermost $\mathrm{C}$ atom at the edge taken from Ref. [44]. Our calculated spectra of edge carbon atoms are similar to calculated results in Ref. [44] and [45]. We 


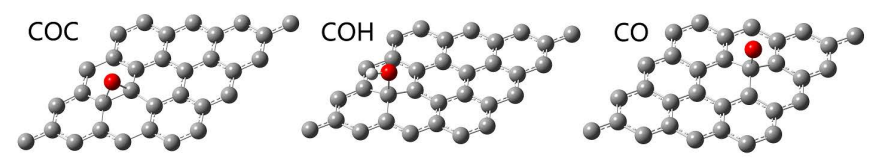

FIG. 11. Supercells of three types of GO, gray - carbon, red - oxygen, white - hydrogen.

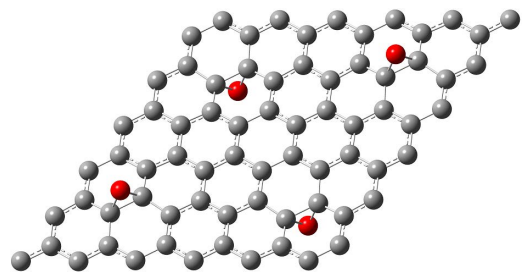

FIG. 12. The supercell used for the calculation of COCtype graphene oxide in the high-density model (STR2) with $\mathrm{C}$ atoms in gray and $\mathrm{O}$ atoms in red.

found that XANES of the C atom labelled by "6" is quite similar to XANES of infinite graphene, except that the whole spectrum is shifted a little to lower energy. However, supercell calculations with vacuum space can not reproduce the absolute value of potential with respect to the vacuum level precisely for low dimensional systems, so that the accuracy of chemical shift between different structures is not ensured.

The overall spectral shape of the experimental spectrum, and in particular the edge-induced low-energy peak, are reproduced by the calculation. The main difference with the spectra of infinite graphene is the appearance of a new strong low-energy peak at $283.8 \mathrm{eV}$, which fits well the pre-edge peak (A2) position observed in some XANES experiments of graphene (see Table III and Fig. 4(a)), and has been attributed to a localized state at the Fermi level at the zigzag edge [46]. Experimentally, Ref. [43] and [47] have found that the more edge $\mathrm{C}$ atoms are probed in XAS, the higher the intensity of the pre-edge peak. Moreover, Other kinds of structure modulations like charged-specie adsorption may also create such a pre-edge peak [43, 48, 49]. From all the experimental and theoretical evidences, it seems that the A2 peak is largely due to edge states or other structural modifications rather than being an intrinsic peak of perfect graphene.

There are some discrepancies of the peak positions between calculated and experimental spectrum of the outermost edge C atom (labelled by "1"), which is also true for another experiment [45]. The disagreement can be attributed to the following points: (i) Core-hole effects are not considered well as described in Sec. III. (ii) The potential generated by VASP code may lead to some deviations, e.g., of vacuum level, since the asymptotic behavior may be not reproduced well for low-dimensional systems in particular directions by the plane-wave method. (iii) The energy resolution of the experiment is $0.4 \mathrm{eV}$ [44], which is not very fine. (iv) The absolute energy of cal-

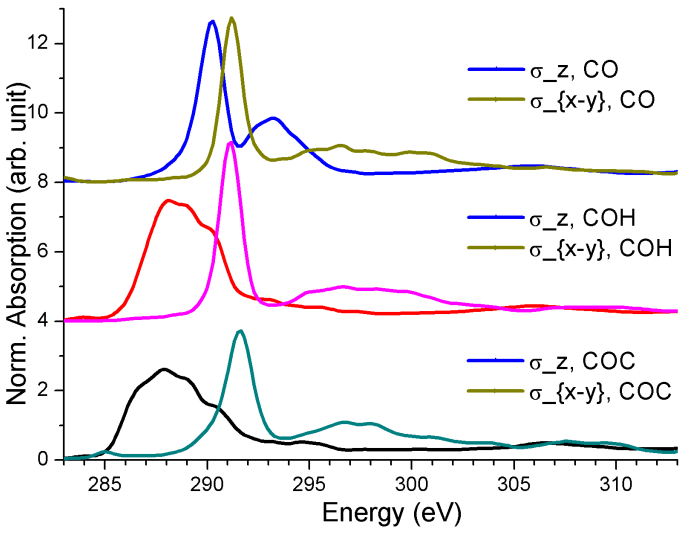

FIG. 13. Calculation of polarization dependent C K-edge XANES of carbon atoms directly bonded to oxygen, for three types of graphene oxide.

culated spectrum is aligned according to experimental XANES of graphene from Ref. [25]. It is not surprising that there is ambiguity in the absolute energies of different experiments. For example, in Ref. [45], the energy position of peak $\pi^{*}$ is fixed as $285.0 \mathrm{eV}$ which is different from $285.5 \mathrm{eV}$ [25] used in this work. (v) In reality the edge structure is not as perfectly ordered as of calculation, it can be distorted or not flat, so that the spectrum is modified. Moreover, the edge structure may be unstable under the incident electron beam [44]. (vi) The contribution of neighboring $\mathrm{C}$ atoms may be mixed in [45].

\section{OXYGEN ADSORPTION ON GRAPHENE}

Among the various graphene derivatives, graphene oxide is the most important one. Oxygen can be bound in different ways to $\mathrm{C}$, on the basal plane, on edge or near vacancies, which makes this system quite complex. For simplicity, we focus on three types of basal-plane oxygen species - $\mathrm{C}-\mathrm{O}-\mathrm{C}(\mathrm{COC}), \mathrm{C}-\mathrm{OH}(\mathrm{COH})$ and $\mathrm{C}=\mathrm{O}(\mathrm{CO})$. This is suitable if the basal plane is complete so that there are few vacancies and the contribution of edge oxygen species can be negligible which is the case for large samples.

We compare two structural models - STR1 and STR2, corresponding to low and high oxygen density, respectively. In STR1, a single oxygen is put on the center of large graphene cluster. In STR2, oxygen atoms are put in a periodic array on graphene, with $4 \mathrm{O}$ atoms per $6 \times 6 \times 1$ SC, corresponding to an oxygen density of $5.3 \%$, see Fig 12. The potential is constructed in the same fashion as before, e.g., for STR1, using FSR potentials from a $4 \times 4 \times 1$ SC (see Fig. 11) VASP calculation for sites closer than $5 \AA$ from the absorber and ground-state potential of the infinite graphene sheet for further away atoms. In both structural models, the cluster size of the XANES calculation is $20 \AA$, and the computational parameters 

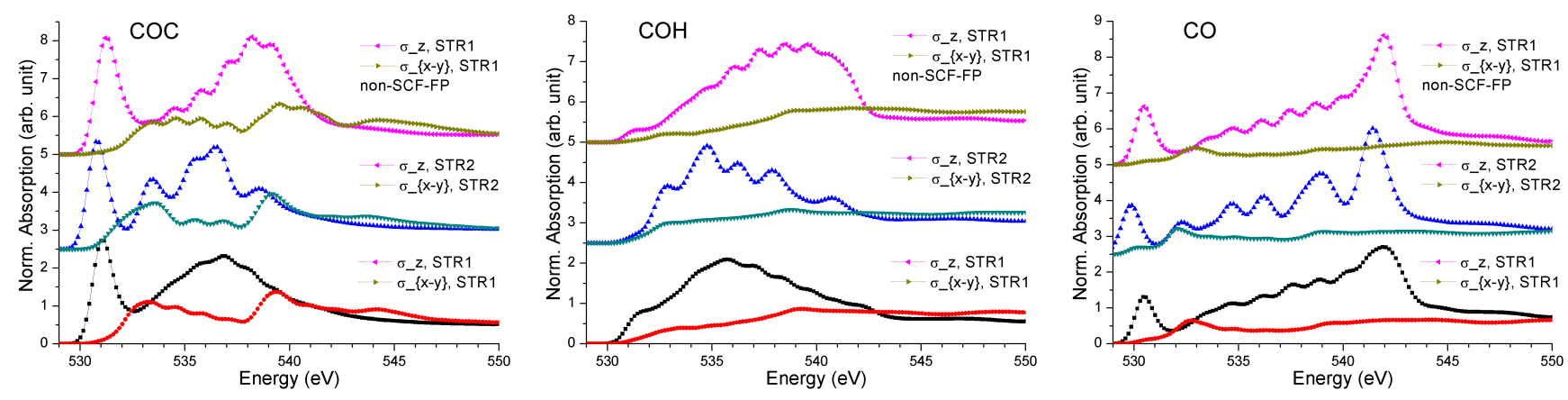

FIG. 14. Calculated O K-edge XANES of GO with different structure models in SCF-FP and non-SCF-FP, STR1 - the structure model containing only one oxygen atom, STR2 - the structure model containing oxygen about $5.3 \%$. Details about structure models are described in Sec. IV.

are similar to the graphene case.

\section{A. C K-edge XANES}

In this section, we consider the low-density structure (STR1) and focus on the XANES spectrum of the C atom directly bonded to oxygen. Firstly, we want to stress that $\mathrm{SCF}$ is important in this case, since in non-SCF-FP calculations ( not shown), the main peaks of $\sigma_{z}$ of COC and $\mathrm{COH}$ are shifted to higher energies by about $1.5 \mathrm{eV}$ compared with SCF-FP results. In Fig. $13, \sigma_{z}$ of COC and $\mathrm{COH}$ have a big peak near 288 and $288.5 \mathrm{eV}$, respectively, which fits the position of peak K. Combining the previous discussions in Sec. III, we make the following conclusions: (i) Since peak K does not arise for ideal graphene and is only weakly affected by stacking of graphene layers, the interpretation using interlayerstate $[25,41]$ is probably not suitable. (ii) Finite size and vacancies in the graphene sheet can lead to some oscillations between the two leading peaks - A and B, which may contribute to peak K. However, for the high-quality graphene sample, this kind of contribution is expected to be quite weak. (iii) Two typical oxygen species of GO adsorbed on the basal plane, $\mathrm{C}-\mathrm{O}-\mathrm{C}$ and $\mathrm{C}-\mathrm{OH}$, give rise to peak K, so that they are two possible origins of peak $\mathrm{K}$ in some experimental XANES of GO.

However, in cases where proportion of oxygen is bigger, other structure modulations can play important roles. In a considerable number of experiments [37, 50-53], near $288 \mathrm{eV}$, we can observe a very pronounced and sharp peak at a higher energy and one or two broader peaks at lower energies. The broader peaks can be attributed to $\mathrm{COC}$ and $\mathrm{COH}$ bondings on the basal plane, while the sharp one is normally attributed to the carboxylic acid group $(-\mathrm{COOH})$ on the edge or near vacancies.

\section{B. O K-edge XANES}

In the MT approximation, the potential in the interstitial region is approximated by a constant. The choice of this parameter leads to an uncertainty in the energy scale and thus in chemical shifts. This ambiguity is absent in FPMS with space-filling cells, and as a result, chemical shifts become predictive and much more accurate than in the MT approximation. Further, since the structures of infinite graphene and graphene oxide studied in our work are similar, and we used vacuum spaces of the same size for the supercell calculations, the chemical shift can be accurate.

Figure 14 shows polarized XANES of $\mathrm{COC}, \mathrm{COH}$ and $\mathrm{CO}$ for the two structural models calculated in SCF-FP and for STR1 also in non-SCF-FP. The peak positions change quite strongly between SCF-FP and non-SCF-FP, which means that the use of SCF potential is important in this case. There are also clear differences between the two structural models, indicating substantial interaction between adsorbed oxygen species in the high-density case (STR2) which leads to extra spectra fine structure.

Next, we have tried to simulate the experimental O Kedge XANES of GO in $800{ }^{\circ} \mathrm{C}$ in Ref. [50] shown in Fig. $15(\mathrm{c})$. This experiment was performed at $90^{\circ}$ incidence of the linearly polarized X-rays so that $\sigma_{z}$ should not contributed to the observed XANES. However, in can be seen in the left picture of Fig. 7 of this same Ref. [50] that the $1 \mathrm{~s}-\pi^{*}$ peak is fairly intense, which implies a nonnegligible $\sigma_{z}$ contribution. By fitting to the experimental spectrum, we find the weight of $\sigma_{z}$ of about $12 \%$.

Cross sections of three oxygen species of two structure models are shown in Fig. 15(a) and 15(b). We have fitted the proportions of $\mathrm{COC}, \mathrm{COH}$ and $\mathrm{CO}$ to the experiment, and obtain $44 \%, 56 \%$ and $0 \%$ for STR1, and $33 \%, 67 \%$ and $0 \%$ for STR2. The final XANES of both structural models are shown in Fig. 15(c). Since the concentration of oxygen is very low, there is an ambiguity of the intensity of the experimental spectra. Therefore, we have done the fittings to its derivative. Both calculations are qualitatively in accordance with the experiment. Moreover, the differences between two calculated results are small. This implies that an oxygen concentration change of several percent will not affect the spectrum much. 

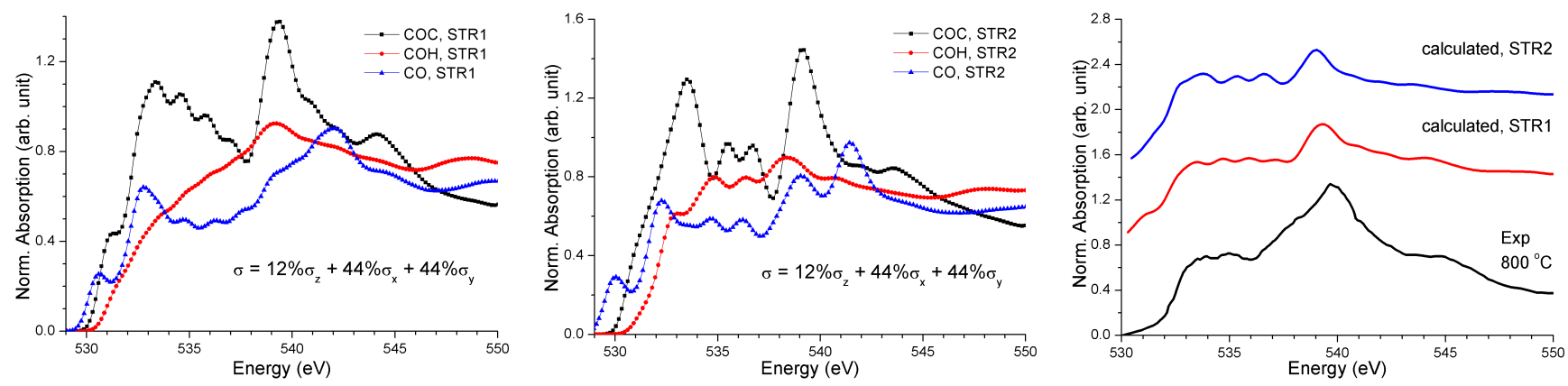

FIG. 15. Calculated O K-edge polarized XANES of GO where the direction of electric field is the same as the experiment [50] and the experiment shown in (b). (a) Using structure mode STR1 containing only one oxygen atom. (b) Using STR2 containing oxygen about $5.3 \%$. (c) The weighted average of XANES of three types of GO using STR1 and STR2. The proportions of $\mathrm{COC}, \mathrm{COH}$ and $\mathrm{CO}$ obtained by fitting them to the experiment are $44 \%, 56 \%$ and $0 \%$ for STR 1 , and $33 \%, 67 \%$ and $0 \%$ for STR2.

\section{COMPARISONS WITH OTHER METHODS}

Here we compare the present FPMS results with three other theoretical methods.

(1) Multiple Scattering in the MT approximation (MTMS). Following common practice, we have used slightly overlapping spheres in the MT calculation. Here the diameter of the spheres is taken $15 \%$ larger than the nearest neighbor distance, i.e., the overlap factor (ovlp) is 1.15. Note that in FPMS, overlap between atomic cells is strictly zero, and when an overlap factor is indicated, it refers to the spheres in which the atomic cells are enclosed.

The spectra in Fig. 16, are plotted on the photoelectron final energy scale, without alignment to experiment, in order to see the peak shifts between different approximations. The SCF-FP calculation with space filling (bottom curve) gives by far the best results (see also Fig. 4). All other schemes, including SCF-FP without ECs, lack most of the fine structure in the energy range $5-20 \mathrm{eV}$. Therefore, both FP treatment inside each atomic cell and filling of interstitial space by empty cells are necessary for accurate XANES. Compared with SCF-FP, all other spectra are shifted, especially those without ECs. This implies that for MS calculations without space-filling, large errors in the absolute energy levels must be expected. Moreover, we have noticed that the results obtained in the MT approximation are very sensitive to the constant interstitial potential and charge, which are usually treated as free parameters.

(2) DFT. In Ref. [5] and [56], Augmented Plane Wave method and pseudopotential plane wave method were used to calculate EELS of graphene, respectively. Their results are similar to ours. In Ref. [57], the authors have done DFT calculations in low energy region with linear combination of atomic orbitals (LCAO) method to obtain initial and final state wavefunctions to compute XAS of graphene. There are some differences between their and our results, e.g. related to layer effects. A drawback of the LCAO method is that uniform convergence of the types and amount of basis functions are not ensured $[58,59]$ over the XANES energy range. This may lead to difficulties for studying XAS in graphene-related systems.

3) PAW Green's function method (PAW-GF). In Ref. [54], a scheme for XANES has been devised based on the recursion method. An advantage of this method is that the Hamiltonian needs to be diagonalized only for occupied states while the core-level spectrum is computed as a continued fraction. In Fig. 17, C K-edge XANES of diamond calculated with PAW-GF [54] and our SCF-FPMS methods are compared with experiment. The two theoretical spectra are very close. This may be expected since the charge densities are computed in the same way (PAW with core- hole in supercell). The almost excellent agreement of the two spectra then clearly shows that in SCFFPMS, potential generation and the change of boundary

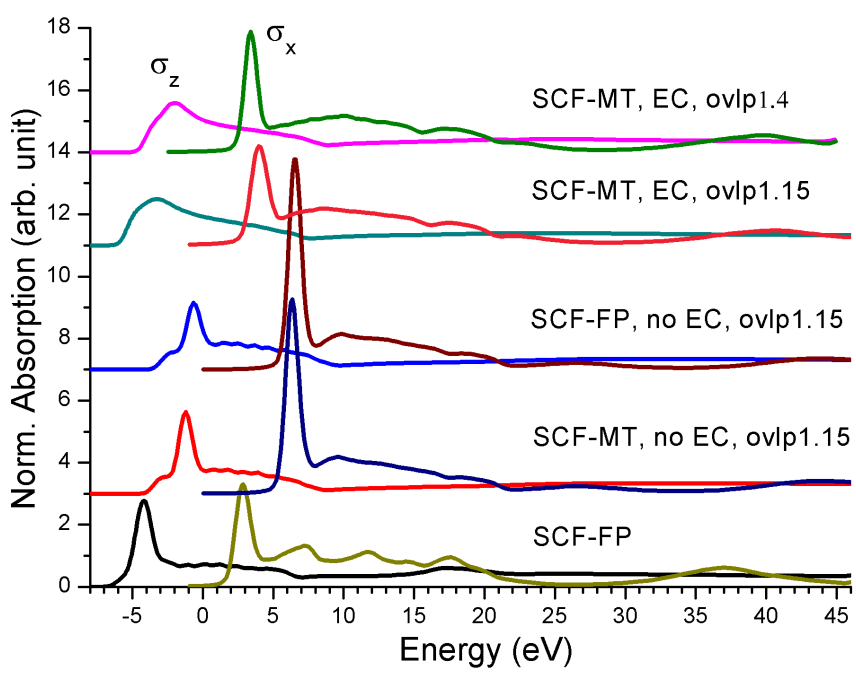

FIG. 16. Calculated XANES of graphene in SCF-FP and SCF-MT cases. The cluster size is always $25 \AA$. ovlp is overlap factor of spherical radii. (no EC) EC represents that ECs are (not) added. 


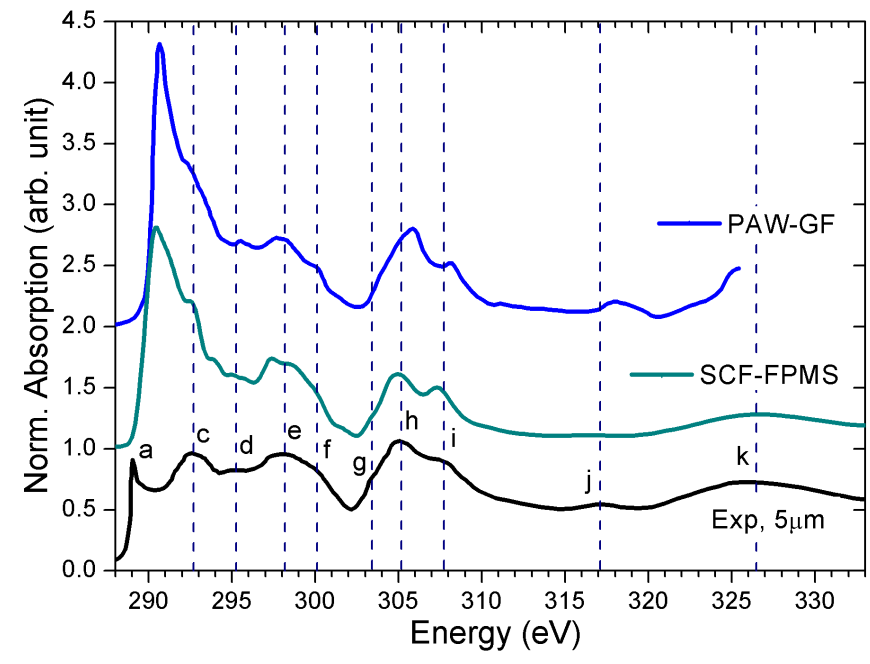

FIG. 17. Calculated C K-edge XANES of diamond by PAWGF [54] and our SCF-FPMS methods compared with experiment from Ref. [55].

conditions from a k- to a real-space cluster method, do not reduce the accuracy of the underlying PAW electronic structure calculation. This is an important check of our new method. While XANES calculations are numerically lighter in PAW-GF, a main advantage of SCF-FPMS is that it can directly be used for a variety of other electron spectroscopies (EXAXS, UPS, XPS, etc) while this remains to be shown for PAW-GF. Further, when looking at the spectra of Fig. 17 in more detail, the SCF-FP spectrum compares slightly better with the experimental one beyond $302 \mathrm{eV}$. This might be due to the fact that in the PAW method the partial waves and projectors are fixed and adapted to the energy interval of the occupied valence electrons. This is not an issue in SCFFPMS, since the all-electron potential is used for solving the Schrödinger equation of the continuum states.

\section{CONCLUSION}

We have presented full-potential MS calculations for XANES of graphene and related systems. The potentials were generated from self-consistent charge densities obtained through plane-wave PAW calculations with the VASP code. With this new scheme $\mathrm{C}$ and $\mathrm{O} \mathrm{K}$ edge XANES have been studied for graphene, few-layer graphene, graphite, three types of graphene oxide, and zigzag edge of graphene, and results have been compared with experiment and several other theoretical methods. With the present full-potential method, good agreement with experiment has been obtained for all systems, while conventional MT calculations give poor results. The main variations of the XANES spectra as a function of the number of graphene layers has been successfully reproduced. The analysis of C K-edge XANES in graphene edge atoms and that of graphene oxide has helped to clarify the origin of several controversial peaks in the graphene spectrum, especially the pre-edge shoulder (A2) and the so-called interlayer-state peak (K). By calculating the $\mathrm{O}$ K-edge XANES of three types of graphene oxide, we showed that the proportion of different oxygen species can be estimated by a fit to the experimental spectrum.

\section{ACKNOWLEDGMENTS}

We would like to thank T. Konishi and W. Huang for illuminating discussions. Computational resources of USTC supercomputing center are gratefully acknowledged. Parts of this work have been funded by European FP7 MSNano network under Grant Agreement No. PIRSES-GA-2012-317554, by FP7 MS-BEEM under Grant Agreement No. FP7-PEOPLE-2013-IEF-625388, by COST Action MP1306 EUSpec and by JSPS KAKENHI Grant Number 25887008.
[1] A. Neto, F. Guinea, N. Peres, K. S. Novoselov, and A. K. Geim. Reviews of modern physics, 81(1):109, 2009.

[2] L. S. Zhang, X. Q. Liang, W. G. Song, and Z. Y. Wu. Phys Chem Chem Phys, 12(38):12055-9, 2010.

[3] D. Haberer, D. V. Vyalikh, S. Taioli, B. Dora, M. Farjam, J. Fink, D. Marchenko, T. Pichler, K. Ziegler, S. Simonucci, M. S. Dresselhaus, M. Knupfer, B. Buchner, and A. Gruneis. Nano Lett, 10(9):3360-6, 2010.

[4] S. Bhowmick, J. Rusz, and O. Eriksson. Physical Review B, 87(15), 2013.

[5] G. Bertoni, L. Calmels, A. Altibelli, and V. Serin. Physical Review B, 71(7), 2005.

[6] Q. Li, Y. Ma, A. Oganov, H. Wang, H. Wang, Y. Xu, T. Cui, H. K. Mao, and G. Zou. Physical Review Letters, 102(17), 2009.

[7] K. Hatada, K. Hayakawa, M. Benfatto, and C. R. Natoli. J Phys Condens Matter, 22(18):185501, 2010.
[8] K. Hatada, K. Hayakawa, M. Benfatto, and C. R. Natoli. Physical Review B, 76(6):060102, 2007.

[9] P. E. Blöchl. Physical Review B, 50(24):17953-17979, 1994.

[10] A. R. Williams et al. Journal of Physics C: Solid State Physics, 7(1):37, 1974.

[11] A. Gonis and W. H. Butler. Multiple scattering in solids. Springer, 2000.

[12] C. R. Natoli, M. Benfatto, C. Brouder, M. López, and D. Foulis. Physical Review B, 42(4):1944-1968, 1990.

[13] D. L. Foulis. Exact distorted-wave approach to multiplescattering theory for general potentials. arXiv preprint arXiv:0806.0574, 2008.

[14] A. L. Ankudinov and J. J. Rehr. Physica Scripta, T115:24-27, 2005.

[15] T. Huhne and H. Ebert. Solid State Communications, 109(9):577-582, 1999. 
[16] G. Kresse and D. Joubert. Physical Review B, 59(3):1758-1775, 1999.

[17] V. I. Lebedev. USSR Computational Mathematics and Mathematical Physics, 15(1):44-51, 1975.

[18] L. Hedin. Physical Review, 139(3A):A796, 1965.

[19] T. X. Carroll, J. Hahne, T. D. Thomas, L. J. Saethre, N. Berrah, J. Bozek, and E. Kukk. Physical Review A, 61(4), 2000.

[20] M. Coville and T. Thomas. Physical Review A, 43(11):6053-6056, 1991.

[21] M. Coreno, M. De Simone, K. C. Prince, R. Richter, M. Vondráček, L. Avaldi, and R. Camilloni. Chemical physics letters, 306(5):269-274, 1999.

[22] A. Goldoni, R. Larciprete, L. Gregoratti, B. Kaulich, M. Kiskinova, Y. Zhang, H. Dai, L. Sangaletti, and F. Parmigiani. Applied Physics Letters, 80(12):2165, 2002.

[23] F. Sette, G. Wertheim, Y. Ma, G. Meigs, S. Modesti, and C. Chen. Physical Review B, 41(14):9766-9770, 1990.

[24] J. P. Perdew, J. A. Chevary, S. H. Vosko, K. A. Jackson, M. R. Pederson, D. J. Singh, and C. Fiolhais. Physical Review B, 46(11):6671, 1992.

[25] D. Pacilé, M. Papagno, A. Rodríguez, M. Grioni, and L. Papagno. Physical Review Letters, 101(6), 2008.

[26] M. Papagno, A. Fraile R., Ç. Ö. Girit, J. C. Meyer, A. Zettl, and D. Pacilé. Chemical Physics Letters, 475(46):269-271, 2009.

[27] Y. Ma, P. Skytt, N. Wassdahl, P. Glans, J. Guo, and J. Nordgren. Physical review letters, 71(22):3725, 1993.

[28] F. Mauri and R. Car. Physical review letters, 75(17):3166, 1995.

[29] M. Ishigami, J. H. Chen, W. G. Cullen, M. S. Fuhrer, and E. D. Williams. Nano Lett, 7(6):1643-8, 2007.

[30] P. Krüger and C. R. Natoli. Physical Review B, $70(24): 245120,2004$.

[31] G. Onida, L. Reining, and A. Rubio. Reviews of Modern Physics, 74(2):601, 2002.

[32] J. Vinson, J. J. Rehr, J. J. Kas, and E. L. Shirley. Physical Review B, 83(11):115106, 2011.

[33] W. Olovsson, I. Tanaka, T. Mizoguchi, P. Puschnig, and C. Ambrosch-Draxl. Physical Review B, 79(4):041102, 2009.

[34] E. L. Shirley. Physical Review Letters, 80(4):794-797, 1998.

[35] S. Trickey, F. Müller-Plathe, G. Diercksen, and J. Boettger. Physical Review B, 45(8):4460-4468, 1992.

[36] E. Duplock, M. Scheffler, and P. Lindan. Physical Review Letters, 92(22), 2004.

[37] V. A. Coleman, R. Knut, O. Karis, H. Grennberg, U. Jansson, R. Quinlan, B. C. Holloway, B. Sanyal, and O. Eriksson. Journal of Physics D: Applied Physics, 41(6):062001, 2008.

[38] M. Klintenberg, S. Lebegue, C. Ortiz, B. Sanyal, J. Fransson, and O. Eriksson. J Phys Condens Matter,
21(33):335502, 2009.

[39] M. Abbas, Z. Y. Wu, J. Zhong, K. Ibrahim, A. Fiori, S. Orlanducci, V. Sessa, M. L. Terranova, and I. Davoli. Applied Physics Letters, 87(5):051923, 2005.

[40] H. K. Jeong, H. J. Noh, J. Y. Kim, L. Colakerol, P. A. Glans, M. Jin, K. Smith, and Y. Lee. Physical Review Letters, 102(9), 2009.

[41] V. N. Strocov, P. Blaha, H. I. Starnberg, M. Rohlfing, R. Claessen, J. M. Debever, and J. M. Themlin. Physical Review B, 61(7):4994-5001, 2000.

[42] R. Rosenberg, P. Love, and V. Rehn. Physical Review B, 33(6):4034-4037, 1986.

[43] B. J. Schultz, C. J. Patridge, V. Lee, C. Jaye, P. S. Lysaght, C. Smith, J. Barnett, D. A. Fischer, D. Prendergast, and S. Banerjee. Nat Commun, 2:372, 2011.

[44] K. Suenaga and M. Koshino. Nature, 468(7327):10881090, 2010.

[45] J. H. Warner, Y. C. Lin, K. He, M. Koshino, and K. Suenaga. Nano letters, 14(11):6155-6159, 2014.

[46] K. Nakada, M. Fujita, G. Dresselhaus, and M. S. Dresselhaus. Phys Rev B Condens Matter, 54(24):17954-17961, 1996.

[47] S. Entani, S. Ikeda, M. Kiguchi, K. Saiki, G. Yoshikawa, I. Nakai, H. Kondoh, and T. Ohta. Applied Physics Letters, 88(15):153126, 2006.

[48] X. Liu, T. Pichler, M. Knupfer, J. Fink, and H. Kataura. Physical Review B, 70(20), 2004.

[49] A. Preobrajenski, M. Ng, A. Vinogradov, and N. Mårtensson. Physical Review B, 78(7), 2008.

[50] A. Ganguly, S. Sharma, P. Papakonstantinou, and J. Hamilton. Journal of Physical Chemistry C, 115(34):17009-17019, 2011.

[51] H. K. Jeong, H. J. Noh, J. Y. Kim, M. H. Jin, C. Y. Park, and Y. H. Lee. EPL (Europhysics Letters), 82(6):67004, 2008.

[52] V. Lee, L. Whittaker, C. Jaye, K. M. Baroudi, D. A. Fischer, and S. Banerjee. Chemistry of Materials, 21(16):3905-3916, 2009.

[53] S. Banerjee, T. Hemraj-Benny, M. Balasubramanian, D. A. Fischer, J. A. Misewich, and S. S. Wong. Chem Commun (Camb), (7):772-3, 2004.

[54] M. Taillefumier, D. Cabaret, A. M. Flank, and F. Mauri. Physical Review B, 66(19), 2002.

[55] Y. K. Chang, H. H. Hsieh, W. F. Pong, M. H. Tsai, F. Z. Chien, P. K. Tseng, L. C. Chen, T. Y. Wang, K. H. Chen, D. M. Bhusari, et al. Physical review letters, 82(26):5377, 1999.

[56] J. Lu, S. P. Gao, and J. Yuan. Ultramicroscopy, 112(1):61-68, 2012.

[57] W. Hua, B. Gao, S. Li, H. Ågren, and Y. Luo. Physical Review B, 82(15), 2010.

[58] M. Iannuzzi and J. Hutter. Phys Chem Chem Phys, 9(13):1599-610, 2007.

[59] B. Brena, S. Carniato, and Y. Luo. J Chem Phys, 122(18):184316, 2005. 